Erratum to: Gestational Trophoblastic Neoplasia, Version 2.2019, NCCN Clinical Practice Guidelines in Oncology: J Natl Compr Canc Netw 2019;17(11):1374-1391.

In the November 2019 issue, the NCCN Clinical Practice Guidelines in Oncology (NCCN Guidelines) for Gestational Trophoblastic Neoplasia, Version 2.2019 (J Natl Compr Canc Netw 2019, doi: 10.6004/jnccn.2019.0053) contained an error.

In 2 places, "hypoglycosylated" should have read "hyperglycosylated." On page 1378, the second sentence under the heading "Follow-up" should have read: "Therefore, monitoring should be performed with a quantitative assay capable of detecting all forms of hCG, including beta-hCG, core hCG, nicked-free beta, beta core, and hyperglycosylated forms. $4,18,19 "$ On page 1380, the last sentence of the second paragraph in the left column should have read: "Elevated hCG with normal hyperglycosylated hCG may indicate quiescent GTN not requiring immediate or further treatment. ${ }^{33 "}$

The online version has been corrected. The authors apologize for the error.

doi: $10.6004 /$ jnccn. 2019.0100

Erratum to: Goulart et al. Lung cancer screening with low-dose computed tomography: costs, national expenditures, and cost-effectiveness. J Natl Compr Canc Netw 2012;10(2):267-275.

The February 2012 article by Goulart et al titled, "Lung Cancer Screening With Low-Dose Computed Tomography: Costs, National Expenditures, and CostEffectiveness," (J Natl Compr Canc Netw 2012, doi: 10.6004/jnccn.2012.0023) was published with an error.

On page 267, in the last sentence in the right-hand column, a percentage given as a "high false-positive screening rate" from the National Lung Screening Trial (NLST) was actually a "high false discovery rate" in the NLST study. Thus, the sentence should have read: "The main concerns include the financial burden that a national [lowdose chest $\mathrm{CT}$ ] screening program would imposed on the U.S. health care system, which currently struggles to contain escalating expenditures, and the patient burden that results from a high false discovery screening rate (estimated as $96.4 \%$ in the NCLS), including unnecessary costs and harms cause by additional imaging tests and surgical procedures."

The journal apologizes for the error. doi: 10.6004/jnccn.2019.0101

\title{
Erratum to: NCCN Guidelines Insights: Small Cell Lung Cancer, Version 2.2018: J Natl Compr Canc Netw 2018;16(10):1171-1182.
}

In the October 2018 issue of JNCCN, the NCCN Guidelines Insights for Small Cell Lung Cancer, Version 2.2018 (J Natl Compr Canc Netw 2018, doi: 10.6004/jnccn.2018.0079) was published with an error.
The name of one of the authors, Jacob Sands, MD, was inadvertently omitted. Dr. Sands' affiliation is Dana-Farber/ Brigham and Women's Cancer Center.

This has been corrected online. The journal apologizes for the error.

doi: $10.6004 /$ jnccn.2019.0102 